\title{
L’acromegalia nell'anziano
}

\author{
Irene Gagliardi ${ }^{1}$ Sabrina Chiloiro ${ }^{2}$ - Antonella Giampietro ${ }^{2}$ - Antonio Bianchi ${ }^{2} \cdot$ Maria Chiara Zatelli $^{1}$. \\ Maria Rosaria Ambrosio ${ }^{1}$
}

Sommario Un aumento del numero di pazienti acromegalici anziani è atteso nei prossimi anni grazie ai miglioramenti apportati in campo diagnostico e terapeutico. La diagnosi di acromegalia nell'anziano può risultare insidiosa a causa di quadri clinici sfumati che possono sovrapporsi alle manifestazioni tipiche dell'invecchiamento. Non esistono criteri diagnostici specifici per l'anziano. La chirurgia si è dimostrata efficace e relativamente sicura anche nel paziente anziano che, tuttavia, presenta una buona risposta agli analoghi della somatostatina.

Parole chiave Acromegalia · Anziano · Comorbidità . Diagnosi · Trattamento

\section{Introduzione}

L'acromegalia è una patologia rara caratterizzata da un'ipersecrezione di ormone della crescita $(\mathrm{GH})$, generalmente dovuta a un adenoma ipofisario GH-secernente, e dalla conseguente iperproduzione epatica del fattore di crescita insulino-simile di tipo 1 (IGF-1) [1]. L'esposizione cronica dell'organismo all'eccesso di GH/IGF-1 induce multiple

Proposta da Maria Rosaria Ambrosio.

Informazioni Supplementari La versione online contiene materiale supplementare disponibile su https://doi.org/10.1007/s40619-021-00864-x.

$凶$ M.R. Ambrosio

mbrmrs@unife.it

1 Sezione di Endocrinologia, Geriatria e Medicina Interna, Dipartimento di Scienze Mediche, Università di Ferrara, Ferrara, Italia

2 U.O. di Patologia Ipotalamo-Ipofisaria, Dipartimento di Endocrinologia, Fondazione A. Gemelli, IRCCS, Università Cattolica del Sacro Cuore, Roma, Italia comorbidità sistemiche. Pertanto, se non trattata, l'acromegalia si associa a una maggiore mortalità, superiore anche di 2-3 volte rispetto a quella della popolazione generale [2]. I recenti progressi nel trattamento dell'acromegalia e delle sue comorbidità, tuttavia, hanno prolungato l'aspettativa di vita del paziente acromegalico [3]. Parallelamente, anche la sopravvivenza della popolazione generale sta aumentando e ciò aumenta la probabilità di nuove diagnosi di acromegalia anche in età più avanzate. Il numero di pazienti acromegalici anziani (con diagnosi de novo o in remissione/buon controllo di malattia) è quindi destinato ad aumentare nei prossimi anni, ma gli studi in letteratura volti a caratterizzare questa particolare categoria di pazienti sono ancora piuttosto scarsi, in assenza di un' uniformità nella definizione di cut-off di anzianità.

In questa rassegna riportiamo le conoscenze ad oggi disponibili sull'epidemiologia, la presentazione clinica, la diagnosi e il trattamento dell' acromegalia nel paziente anziano.

\section{Epidemiologia}

Ad oggi non sono disponibili studi epidemiologici specifici per la popolazione acromegalica anziana. I dati derivati da recenti analisi condotte sulla popolazione acromegalica in toto mostrano un aumento di prevalenza dell' acromegalia nelle fasce d'età maggiori. Nella popolazione statunitense, Broder e collaboratori hanno rilevato valori di prevalenza maggiori tra i pazienti con età compresa tra i 55 e i 64 anni (11,5 casi per 100.000 abitanti) [4]. Burton e colleghi hanno stimato una prevalenza di $14,8-18,2$ per 100.000 abitanti nella popolazione over 65 [5]. I dati italiani confermano una popolazione acromegalica composta quasi per la metà $(46 \%)$ da pazienti over 65 , rientranti perlopiù nella settima decade [6]. Petrossian e collaboratori hanno osservato in 
Fig. 1 Caratteristiche cliniche del paziente acromegalico anziano

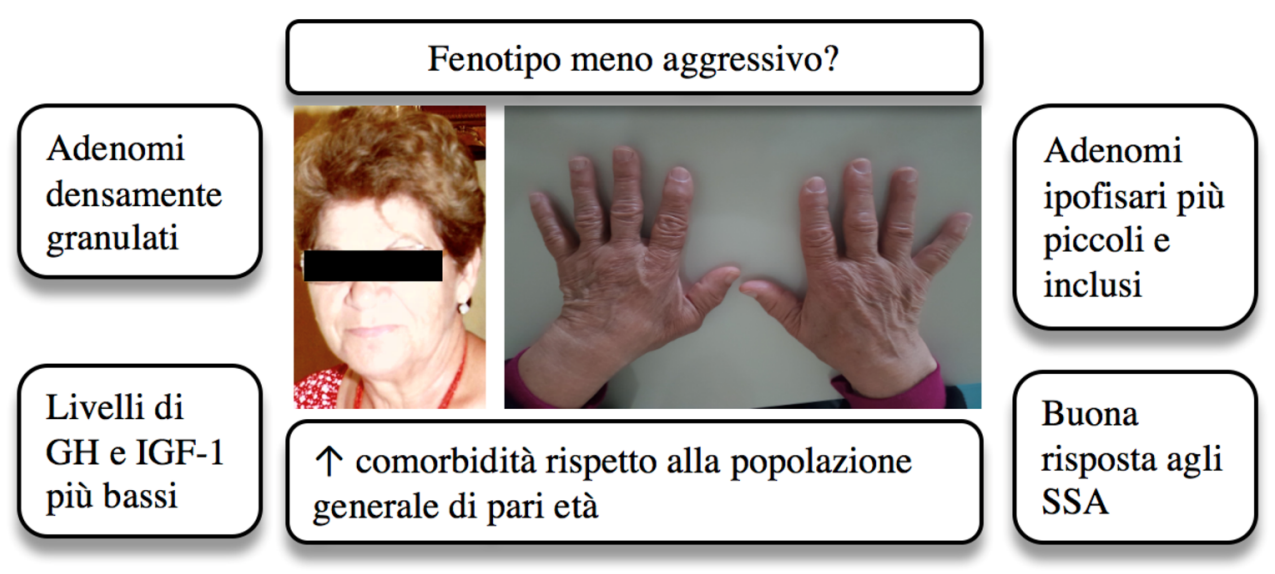

3.173 pazienti appartenenti a 10 diversi paesi un progressivo aumento dell'età media alla diagnosi (41,8 anni prima del 1990 vs 48,79 dopo il 2010) in entrambi i sessi, con una parallela riduzione del ritardo diagnostico [7]. Anche il tasso di incidenza dell'acromegalia ha mostrato un'associazione positiva con l'età $(0,3-0,8$ casi per 100.000 abitanti 0-17 anni; 0,9-1,8 casi per $100.000>65$ anni) nella popolazione statunitense [5]. Lo studio retrospettivo di Arosio e colleghi, condotto su una casistica italiana, ha evidenziato nuove diagnosi di acromegalia in circa il $3 \%$ dei maschi e $5 \%$ delle femmine $>65$ anni e ha dimostrato come nei pazienti con malattia controllata $(65 \%)$ il tasso standardizzato di mortalità non fosse aumentato. La mortalità, al contrario, si manteneva elevata solo in quei pazienti con malattia attiva, a dimostrazione di come il trattamento dell'acromegalia e il buon controllo della malattia possano influire positivamente sulla sopravvivenza [8]. L'aumentata aspettativa di vita ha determinato anche un cambiamento delle cause di decesso, che oggigiorno sembrano più simili a quelle della popolazione generale di pari età. Se prima del 2004, infatti, gli studi riportavano come principale causa di morte del paziente acromegalico le patologie cardiovascolari, attualmente la patologia neoplastica sembra la più frequente causa di decesso [3, 9].

\section{Presentazione clinica}

La diagnosi di acromegalia nel paziente anziano può risultare insidiosa poiché le tipiche modificazioni somatiche si instaurano lentamente, contribuendo a quadri clinici sfumati che spesso si sovrappongono alle manifestazioni tipiche dell'invecchiamento $[6,10]$. Ciò può aumentare il rischio di ritardo diagnostico in questa categoria di pazienti, calcolato fino a 12,3 anni in pazienti $>50$ anni, rispetto a un ritardo di 6 anni nei pazienti $<31$ anni, di 7 anni nei pazienti tra i 31 e i 40 anni e di 10,2 anni nei pazienti tra i 41 e i 50 anni [11]. Pochi studi in letteratura hanno descritto i segni e i sintomi

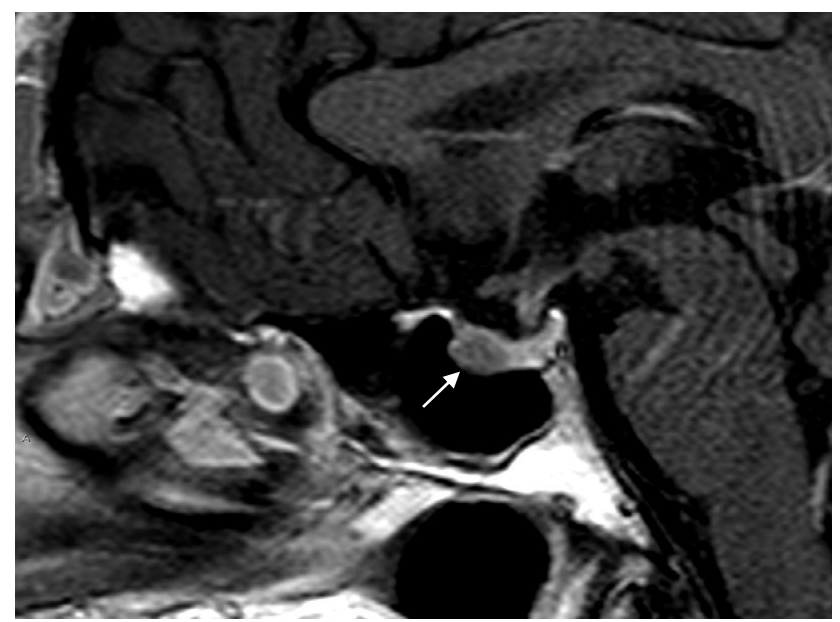

Fig. 2 Risonanza Magnetica sellare T1 sagittale con mdc: adenoma ipofisario GH secernente incluso nel cavo sellare

specifici del paziente acromegalico anziano alla diagnosi e in pochi è stato eseguito il confronto con un gruppo di controllo. L'acromegalico anziano sembra presentare un fenotipo meno aggressivo (Fig. 1), a causa di adenomi ipofisari più piccoli e inclusi nel cavo sellare (Fig. 2) e/o alla presenza di più bassi livelli di GH/IGF-1 [12]. Alla diagnosi possono essere già presenti ipertensione, anomalie metaboliche, artralgie, gozzo tiroideo [13]. Dalla valutazione di serie chirurgiche di pazienti over 65 sono stati registrati inoltre: ipopituitarismo, habitus acromegaloide, ingrandimento acrale, macroglossia, iperidrosi, artralgia, cefalea, perdita del visus [14]. Gli studi dedicati alla ricerca di comorbidità croniche nella popolazione acromegalica anziana sono piuttosto limitati. L'età stessa potrebbe essere un fattore confondente, dal momento che rappresenta un fattore di rischio per lo sviluppo delle medesime comorbidità anche nella popolazione anziana generale (Tabella 1) [6].

Nell'acromegalia l'età sembra rappresentare un fattore di rischio per lo sviluppo di patologie cardiovascolari [6]. In uno studio di Colao e collaboratori, l'indice di massa ventri- 
Tabella 1 Comorbidità nei pazienti acromegalici anziani

Comorbidità

Ipertensione, alterazione del metabolismo glucidico, complicanze articolari, gozzo tiroideo

Ipertrofia ventricolare sinistra

Artropatia e rischio di frattura vertebrale $\rightarrow$

$\rightarrow \quad$ Caratteristica del paziente acromegalico anziano

Non sono correlate all'età colare sinistra aumentava in maniera significativa con l'età e la prevalenza di ipertrofia ventricolare sinistra era maggiore nei pazienti $>50$ anni $(74,3 \%)$ rispetto ai più giovani (35-37\%). Inoltre, l'ipertensione gioca un ruolo significativo nello sviluppo dell'ipertrofia cardiaca, soprattutto nei pazienti anziani [13].

È stato osservato come l'età predisponga a un maggior rischio di alterato metabolismo glucidico nell'acromegalia rispetto alla popolazione sana, unitamente alla presenza di altri fattori di rischio quali una lunga durata di malattia e la familiarità per diabete. Le evidenze relative alle alterazioni del metabolismo lipidico in rapporto all'età nei pazienti acromegalici anziani, invece, sono ancora contrastanti [6].

La prevalenza di OSAS nella popolazione acromegalica anziana sembra essere simile a quella della popolazione acromegalica più giovane [6].

La frequenza di problematiche reumatologiche (artralgia, tunnel carpale o osteoartriti) non differisce in maniera significativa rispetto alla popolazione anziana non acromegalica. I dati ad oggi a disposizione, inoltre, suggeriscono come l'età non sia un fattore predittivo di rischio per frattura vertebrale da fragilità nei pazienti acromegalici anziani [6].

Il cancro sembra essere diventata una patologia etàdipendente anche nell'acromegalia e la sua aumentata incidenza potrebbe essere spiegata da una maggiore longevità di questi pazienti. Inoltre, alcune delle comorbidità acromegalia-relate, ad esempio l'obesità e il diabete, sono noti fattori di rischio per lo sviluppo di neoplasie [3].

Infine, nei pazienti acromegalici anziani le funzioni neuro-cognitive e psicologiche, la performance fisica e il grado di indipendenza nelle attività quotidiane sembrerebbero maggiormente compromesse rispetto alla popolazione sana di pari età [15].

\section{Diagnosi}

Non esistono criteri diagnostici di remissione/controllo di malattia specifici per la popolazione acromegalica anziana. L'età potrebbe, tuttavia, correlare negativamente con i livelli di GH/IGF-1 anche nel paziente anziano acromegalico, così come nella popolazione generale. La letteratura documenta ampiamente una relazione inversa tra livelli basali di GH alla diagnosi/nadir dopo carico orale di glucosio con l'età
$[7,16]$. L'esecuzione di una curva da carico orale di glucosio a scopo diagnostico potrebbe non essere sempre applicabile nella popolazione geriatrica per la coesistenza di diabete: in tal caso è possibile eseguire prelievi seriati per GH. Anche nei pazienti anziani è indicato l'approfondimento radiologico con RM sellare con mdc, previa conferma di una funzione renale conservata [6].

\section{Istopatologia}

Gli adenomi ipofisari GH-secernenti sono classificati sulla base della densità intracellulare dei granuli secretori in adenomi densamente granulati (DG) e sparsamente granulati (SG). Questi ultimi sono stati associati a una maggiore aggressività, oltre che a età più precoci di diagnosi, mentre gli adenomi DG sembrano più frequenti nei pazienti anziani. Tali associazioni rimangono, tuttavia, ancora speculative e ulteriori studi di confronto tra pazienti di diversi gruppi di età sono necessari [5].

\section{Trattamento}

Gli obiettivi di trattamento nei pazienti anziani sono simili a quelli dei pazienti più giovani e includono il controllo ormonale di malattia, la riduzione della massa ipofisaria e la gestione delle comorbidità [17]. Anche nei pazienti anziani la chirurgia TNS rappresenta la prima linea di trattamento con un basso rischio di morbidità e un'alta probabilità di remissione, soprattutto per i microadenomi e i macroadenomi inclusi. Rispetto ai pazienti più giovani, gli acromegalici anziani non sembrano manifestare un più alto rischio di complicanze perioperatorie. Pertanto, la chirurgia TNS può essere considerata come un trattamento sicuro se preceduta da un' accurata valutazione del rischio anestesiologico/chirurgico [18]. Tuttavia, occorre considerare come gli anziani siano più suscettibili allo stress da ospedalizzazione e come la presenza di ipertensione, patologie cardiovascolari e diabete mellito aggravino la vulnerabilità di questa popolazione [6]. I pazienti acromegalici anziani, soprattutto di sesso maschile, mostrano una migliore risposta biochimica all'octreotide [19]. Tale dato rinforza l'ipotesi di una terapia medica 
con analoghi della somatostatina (SSA) di prima generazione come trattamento medico di prima scelta nella popolazione anziana, in assenza di effetti collaterali severi nel lungo termine [6]. Pasireotide può rappresentare un'alternativa in caso di resistenza alla terapia con SSA di prima generazione, a condizione di uno stretto monitoraggio glicemico, dal momento che l'iperglicemia è un effetto collaterale frequente. Non vi sono dati consistenti relativi all'utilizzo del pegvisomant nella popolazione anziana. Tuttavia, non sono note complicanze del pegvisomant tali da limitarne l'utilizzo in età geriatrica. Non vi sono ancora dati circa l'influenza dell'età sulla risposta ai farmaci dopaminergici [6]. Un recente studio retrospettivo italiano ha osservato nella propria coorte di pazienti acromegalici come i pazienti anziani siano stati sottoposti più frequentemente a trattamento medico di prima linea con SSA, con il raggiungimento del controllo biochimico di malattia nel 55\% dei casi. Ulteriori studi prospettici sono tuttavia necessari per valutare l'effettiva efficacia della terapia medica di prima linea nel trattamento dell'acromegalia nel paziente anziano: se da una parte, infatti, può rappresentare un'opzione più sicura con un minor rischio di complicanze rispetto alla chirurgia, dall'altra occorre considerare anche la sostenibilità economica di un trattamento cronico a lungo termine [12]. L'uso della radioterapia nei pazienti anziani è limitato dalla necessità di un tempo prolungato dopo l'irradiazione (dai 5 ai 10 anni) per verificare l'efficacia del trattamento. Inoltre' la radioterapia è associata a un alto rischio di ipopituitarismo con necessità di terapie sostitutive e un potenziale rischio di patologia cerebrovascolare, condizioni che potrebbero limitarne l'utilizzo nelle età più avanzate [6].

\section{Conclusioni}

A fronte in un numero di pazienti acromegalici anziani in aumento nei prossimi anni, i dati di letteratura sono ancora sporadici e non conclusivi nel definirne le caratteristiche epidemiologiche, fisiopatologiche, cliniche, diagnostiche e di riposta al trattamento. Ulteriori studi caso-controllo sono pertanto necessari al fine di ampliare le conoscenze su questa categoria di pazienti e di garantire una gestione clinica mirata a rispondere alle peculiari necessità del paziente acromegalico anziano.

Funding Note Open access funding provided by Università degli Studi di Ferrara within the CRUI-CARE Agreement.

Conflitto di interesse Gli autori Irene Gagliardi, Sabrina Chiloiro, Antonella Giampietro, Antonio Bianchi, Maria Chiara Zatelli e Maria Rosaria Ambrosio dichiarano di non avere conflitti di interesse.

Consenso informato Lo studio presentato in questo articolo non ha richiesto sperimentazione umana.
Studi sugli animali Gli autori di questo articolo non hanno eseguito studi sugli animali.

Nota della casa editrice Springer Nature rimane neutrale in riguardo alle rivendicazioni giurisdizionali nelle mappe pubblicate e nelle affiliazioni istituzionali.

Open Access This article is licensed under a Creative Commons Attribution 4.0 International License, which permits use, sharing, adaptation, distribution and reproduction in any medium or format, as long as you give appropriate credit to the original author(s) and the source, provide a link to the Creative Commons licence, and indicate if changes were made. The images or other third party material in this article are included in the article's Creative Commons licence, unless indicated otherwise in a credit line to the material. If material is not included in the article's Creative Commons licence and your intended use is not permitted by statutory regulation or exceeds the permitted use, you will need to obtain permission directly from the copyright holder. To view a copy of this licence, visit http://creativecommons.org/licenses/by/4.0/.

\section{Bibliografia}

1. Colao A, Grasso LF, Giustina A et al (2019) Acromegaly. Nat Rev Dis Primers 5(1):20

2. Wright AD, Hill DM, Lowy C, Fraser TR (1970) Mortality in acromegaly. Q J Med 39(153):1-16

3. Bolfi F, Neves AF, Boguszewski CL, Nunes-Nogueira VS (2018) Mortality in acromegaly decreased in the last decade: a systematic review and meta-analysis. Eur J Endocrinol 179(1):59-71

4. Broder MS, Chang E, Cherepanov D et al (2016) Incidence and prevalence of acromegaly in the United States: a claims-based analysis. Endocr Pract 22(11):1327-1335

5. Burton T, Le Nestour E, Neary M, Ludlam WH (2016) Incidence and prevalence of acromegaly in a large US health plan database. Pituitary 19(3):262-267

6. Ambrosio MR, Gagliardi I, Chiloiro S et al (2020) Acromegaly in the elderly patients. Endocrine 68(1):16-31

7. Petrossians P, Daly AF, Natchev E et al (2017) Acromegaly at diagnosis in 3173 patients from the Liège Acromegaly Survey (LAS) database. Endocr Relat Cancer 24(10):505-518

8. Arosio M, Reimondo G, Malchiodi E et al (2012) Predictors of morbidity and mortality in acromegaly: an Italian survey. Eur J Endocrinol 167(2):189-198

9. Terzolo M, Reimondo G, Berchialla P et al (2017) Acromegaly is associated with increased cancer risk: a survey in Italy. Endocr Relat Cancer 24(9):495-504

10. Jallad RS, Bronstein MD (2019) Acromegaly in the elderly patient. Arch Endocrinol Metab 63(6):638-645

11. Nabarro JD (1987) Acromegaly. Clin Endocrinol (Oxf) 26(4):481-512

12. Ceccato F, Barbot M, Lizzul L et al (2020) Clinical presentation and management of acromegaly in elderly patients. Hormones 20:143-150

13. Colao A, Pivonello R, Spinelli L et al (2007) A retrospective analysis on biochemical parameters, cardiovascular risk and cardiomyopathy in elderly acromegalic patients. J Endocrinol Invest 30(6):497-506

14. Colao A, Amato G, Pedroncelli AM et al (2002) Gender- and age-related differences in the endocrine parameters of acromegaly. J Endocrinol Invest 25(6):532-538

15. Hatipoglu E, Yuruyen M, Keskin E et al (2015) Acromegaly and aging: a comparative cross-sectional study. Growth Horm IGF Res 25(1):47-52 
16. Ribeiro-Oliveira A, Abrantes MM, Barkan AL (2013) Complex rhythmicity and age dependence of growth hormone secretion are preserved in patients with acromegaly: further evidence for a present hypothalamic control of pituitary somatotropinomas. J Clin Endocrinol Metab 98(7):2959-2966

17. Katznelson L, Laws ER, Melmed S et al (2014) Acromegaly: an endocrine society clinical practice guideline. J Clin Endocrinol Metab 99(11):3933-3951
18. Sasagawa Y, Hayashi Y, Tachibana O et al (2018) Transsphenoidal surgery for elderly patients with acromegaly and its outcomes: comparison with younger patients. World Neurosurg 118:e229-234

19. van der Lely AJ, Harris AG, Lamberts SW (1992) The sensitivity of growth hormone secretion to medical treatment in acromegalic patients: influence of age and sex. Clin Endocrinol (Oxf) 37(2):181-185 